This is the accepted version of the article published by Springer Verlag: Bonenti, F., Martínez-Legaz, J.E. (2015) On the Existence of a Saddle Value, Journal of Optimization Theory and Applications, 165(3): 785-792. The final version is available at: https://link. springer.com/article/10.1007/s10957-014-0665-9

\title{
On the Existence of a Saddle Value
}

\author{
F. Bonenti \\ Dipartimento di Economia e Management \\ Università degli Studi di Brescia \\ Contrada Santa Chiara 50, 25122 Brescia, Italy \\ J.E. MARTÍNEZ-LEGAZ* \\ Departament d'Economia i d'Història Econòmica \\ Universitat Autònoma de Barcelona \\ 08193 Bellaterra, Spain
}

Communicated by Stefan Rolewicz

\begin{abstract}
In this work, we achieve a complete characterization of the existence of a saddle value, for bifunctions which are convex, proper and lower semicontinuous in their first argument, by considering new suitably defined notions of special directions of recession. As special cases, we obtain some recent results of Lagrangian duality theory on zero duality gap for convex programs.
\end{abstract}

\section{Introduction}

Investigations on the existence of a saddle value for a given function date back to von Neumann, in the context of game theory. In [1] he proved the connection between this existence and the solvability of systems of linear inequalities.

Since saddle value theorems using convexity assumptions have found important applications in different fields of Mathematics, there have been later studies under generalized convexity assumptions: for example [2] shows the correspondence, in terms of Lagrangians, between the problems that admit a saddle value and convex programs associated with closed, proper and convex bifunctions, while [3] contains some theorems guaranteeing the existence of a saddle value in various topological and non topological situations.

In particular, the minimax theorem for a convex-concave bifunction is a fundamental theorem in optimization and convex analysis, and has various applications in economics. In [4, Sect. 37] Rockafellar gave a characterization of the existence of a saddle value, without considering directions of recession.

*Corresponding author. e-mail: JuanEnrique.Martinez.Legaz@uab.cat 
Actually, the study of bifunctions plays an important role in Variational Analysis and, in particular, in the analysis of the Lagrangian associated with an optimization problem. If the optimal value of the given optimization problem is different from that of its associated Lagrangian dual problem, one says that there is a duality gap. A basic issue in convex programming is that of finding conditions ensuring that there is no duality gap.

Starting with the well-known Clark-Duffin Theorem [5, Thm. 2], which states that for a convex program defined over a Euclidean space there is no duality gap if there is no direction of recession common to both the objective function and the feasible set, the problem of finding conditions ensuring no duality gap, involving directions of recession, has been extensively studied. In [6], Jeyakumar and Wolkowicz gave an infinite dimensional generalization of the Clark-Duffin Theorem. In [7, Sect. 4] Ernst and Volle provided a characterization of the absence of a duality gap for convex programs, in terms of directions of recession, and defined and studied several suitable notions of special directions of recession for closed convex sets and lower semi continuous proper convex functions.

The notion of saddle value of the Lagrangian function is also fundamental: it permits us to treat in a unified way the primal and dual aspects of optimization problems. Note that the existence of a saddle value means that there is no duality gap, and it is possible to have no duality gap without the existence of saddle-points (see [4, Sect. 36]).

In the present paper we extend some results presented in [7], and achieve a complete characterization of the existence of a saddle value, by considering new suitably defined notions of special directions of recession for bifunctions depending on the first argument in a convex, proper and lower semi continuous way.

\section{Preliminaries}

Let $f: \mathbb{R}^{n} \rightarrow \mathbb{R} \cup\{+\infty\}$ be a lower semicontinuous (l.s.c.) proper convex function and $g=\left(g_{1}, \ldots, g_{m}\right): \mathbb{R}^{n} \rightarrow(\mathbb{R} \cup\{+\infty\})^{m}$ be a vector function with l.s.c. proper convex components. The Lagrangian $\mathcal{L}: \mathbb{R}^{n} \times \mathbb{R}_{+}^{m} \longrightarrow \mathbb{R} \cup\{+\infty\}$ of the convex program

$(\mathcal{P}): \quad \quad \inf \left\{f(x): x \in \mathbb{R}^{n}, g(x) \leqslant 0\right\}$

is defined by

$$
\mathcal{L}(x, \lambda):=f(x)+\langle\lambda, g(x)\rangle,
$$

with $\langle\lambda, g(x)\rangle:=\sum_{i=1}^{m} \lambda_{i} g_{i}(x)$ for $\lambda:=\left(\lambda_{1}, \ldots, \lambda_{m}\right)$, using the following convention $0(+\infty):=+\infty$. Throughout this paper, we will always assume that $(\mathcal{P})$ is consistent, that is, its feasible set $\left\{x \in \mathbb{R}^{n}: g(x) \leqslant 0\right\}$ is non-empty.

The convex program $(\mathcal{P})$ is associated with the so called Lagrangian dual problem:

$$
\sup \left\{\inf _{x \in \mathbb{R}^{n}} \mathcal{L}(x, \lambda): \lambda \geqslant 0\right\} .
$$


Let $\Lambda$ be an arbitrary set and $\mathcal{L}: \mathbb{R}^{n} \times \Lambda \longrightarrow \mathbb{R} \cup\{+\infty\}$ be a bifunction. This paper deals with conditions for the equality

$$
\sup _{\lambda \in \Lambda} \inf _{x \in \mathbb{R}^{n}} \mathcal{L}(x, \lambda)=\inf _{x \in \mathbb{R}^{n}} \sup _{\lambda \in \Lambda} \mathcal{L}(x, \lambda)
$$

to hold. In such a case, the common value on both sides of (3) is called the minimax or saddle value of $\mathcal{L}$. Even though this equality does not necessarily hold, one has the following well known result.

Lemma 1.1 [4, Lemma 36.1] For every set $\Lambda$ and for every bifunction $\mathcal{L}: \mathbb{R}^{n} \times \Lambda \longrightarrow \mathbb{R} \cup\{+\infty\}$, one has

$$
\sup _{\lambda \in \Lambda} \inf _{x \in \mathbb{R}^{n}} \mathcal{L}(x, \lambda) \leqslant \inf _{x \in \mathbb{R}^{n}} \sup _{\lambda \in \Lambda} \mathcal{L}(x, \lambda) .
$$

The sufficient conditions we will present for the equality (3) to hold will be expressed in terms of directions of recession. We next recall the notions of directions of recession for sets, functions and optimization problems.

Definition $1.1\left[4\right.$, Thm. 8.1] A vector $v \in \mathbb{R}^{n}$ is a direction of recession of a closed and convex set $C \subseteq \mathbb{R}^{n}$ iff $v+C \subseteq C$.

Definition 1.2 A vector $v \in \mathbb{R}^{n}$ is a direction of recession of a l.s.c. proper convex function $f: \mathbb{R}^{n} \rightarrow \mathbb{R} \cup\{+\infty\}$ iff $f$ is non-increasing over every half-line of direction $v$.

Remark 1.1 According to [4, Thm. 8.6], for $v$ to be a direction of recession of $f$ it is sufficient that it be non-increasing on some half-line of direction $v$.

Let $f: \mathbb{R}^{n} \rightarrow \mathbb{R} \cup\{+\infty\}$ be convex, proper and l.s.c., and assume that the vector-valued function $g: \mathbb{R}^{n} \rightarrow(\mathbb{R} \cup\{+\infty\})^{m}$ has l.s.c. proper convex components. We will consider the convex program $(\mathcal{P})$ defined in $(1)$.

Definition 1.3 A vector $v \in \mathbb{R}^{n}$ is a direction of recession of $(\mathcal{P})$ if and only if it is a direction of recession of both $f$ and the feasible set $\left\{x \in \mathbb{R}^{n}: g(x) \leqslant 0\right\}$.

Proposition 1.1 Let $(\mathcal{P})$ be the consistent convex program defined by (1), let $\mathcal{L}$ be the Lagrangian of $(\mathcal{P})$ defined by $(2)$, and let $v \in \mathbb{R}^{n}$. The following statements are equivalent:

1. $v$ is a direction of recession of $(\mathcal{P})$;

2. $v$ is a direction of recession of $f$ and every component of $g$;

3. $v$ is a direction of recession of $\mathcal{L}(\cdot, \lambda)$ for all $\lambda \in \mathbb{R}_{+}^{m}$.

Proof. $(1 \Rightarrow 2)$. In view of Remark 1.1, we only need to prove that each component $g_{i}$ of $g$ is non-increasing on some half-line of direction $v$. Take a feasible point $x$ of $(\mathcal{P})$, and consider the one variable convex function $\varphi$ defined by $\varphi(t):=g_{i}(x+t v)$. Since $x$ is feasible and $v$ is a direction of recession of the 
feasible set, we have $\varphi(t) \leq 0$ for every $t \geq 0$. Let $0 \leq t_{1}<t_{2}<t$. From the convexity of $\varphi$ it follows that

$$
\varphi\left(t_{2}\right) \leq \frac{t-t_{2}}{t-t_{1}} \varphi\left(t_{1}\right)+\frac{t_{2}-t_{1}}{t-t_{1}} \varphi(t) \leq \frac{t-t_{2}}{t-t_{1}} \varphi\left(t_{1}\right) .
$$

Setting $t \longrightarrow+\infty$, we get $\varphi\left(t_{2}\right) \leq \varphi\left(t_{1}\right)$, which shows that $g_{i}$ is non-increasing on the half-line $x+t v, t \geq 0$.

$(2 \Rightarrow 1)$ and $(2 \Rightarrow 3)$ are obvious by definition.

$(3 \Rightarrow 2)$. Taking $\lambda:=0$ and using Remark 1.1 and the fact that $(\mathcal{P})$ is consistent, it turns out that $v$ is a direction of recession of $f$. Moreover, for every feasible $x$ and every $\lambda \in \mathbb{R}_{+}^{m}$ we have

$$
f^{\prime}(x, v)+\left\langle\lambda, g^{\prime}(x, v)\right\rangle=\mathcal{L}(\cdot, \lambda)^{\prime}(x, v) \leqslant 0 .
$$

It follows that

$$
g^{\prime}(x, v) \leqslant 0,
$$

which, again by Remark 1.1, means that $v$ is a direction of recession of every component of $g$.

The following notions of special directions of recession will play a crucial role in the sequel.

Definition 1.4 [7, Def. 4.3] A vector $v \in \mathbb{R}^{n}$ is an ia-direction of recession of $f$ iff for every $\bar{x} \in \mathbb{R}^{n}$ one has

$$
\liminf _{s \rightarrow+\infty} f(\bar{x}+s v)=\inf _{x \in \mathbb{R}^{n}} f(x) .
$$

We recall that the indicator function $\delta_{C}: \mathbb{R}^{n} \longrightarrow \mathbb{R} \cup\{+\infty\}$ of a set $C \subseteq \mathbb{R}^{n}$ is defined by $\delta_{C}(x):=0$ if $x \in C, \delta_{C}(x):=+\infty$ if $x \in \mathbb{R}^{n} \backslash C$.

Definition 1.5 [7, Def. 4.4] A vector $v \in \mathbb{R}^{n}$ is an ia-direction of recession of a non-empty closed and convex set $C \subseteq \mathbb{R}^{n}$ iff it is an ia-direction of recession of its indicator function $\delta_{C}$.

Remark 1.2 [7, Remark 4.1] A direction of recession $v$ of a non-empty closed and convex set $C$ is an ia-direction of recession if and only if no half-line of direction $v$ lies entirely outside $C$.

\section{Main Results}

To obtain our results, besides the already exisiting notions of special directions of recession, which we have recalled in the preceding section, we need to introduce some new related concepts.

Definition 2.1 A vector $v \in \mathbb{R}^{n}$ is a partial ia-direction of recession of $f$ iff there exists $\bar{x} \in \mathbb{R}^{n}$ such that (4) holds. 
Clearly, every ia-direction of recession is a partial ia-direction of recession.

Definition 2.2 Let $\Lambda$ be an arbitrary non-empty set, and $\mathcal{L}: \mathbb{R}^{n} \times \Lambda \longrightarrow \mathbb{R} \cup\{+\infty\}$ be convex, proper and l.s.c. with respect to its first argument. A vector $v \in \mathbb{R}^{n}$ is a saddle ia-direction of recession of $\mathcal{L}$ relative to its first argument iff there exists $\bar{x} \in \mathbb{R}^{n}$ such that

$$
\liminf _{s \rightarrow+\infty} \sup _{\lambda \in \Lambda} \mathcal{L}(\bar{x}+s v, \lambda)=\sup _{\lambda \in \Lambda} \inf _{x \in \mathbb{R}^{n}} \mathcal{L}(x, \lambda) .
$$

Definition 2.3 Let $\Lambda$ be an arbitrary non-empty set, $\mathcal{L}: \mathbb{R}^{n} \times \Lambda \longrightarrow \mathbb{R} \cup\{+\infty\}$ be convex, proper and l.s.c. with respect to its first argument, and $A$ be a nonempty subset of $\mathbb{R}^{n}$. A vector $v \in \mathbb{R}^{n}$ is an A-saddle ia-direction of recession of $\mathcal{L}$ relative to its first argument if (5) holds for every $\bar{x} \in A$.

Clearly, every $A$-saddle ia-direction of recession is a saddle ia-direction of recession.

The following theorem characterizes saddle ia-directions of recession and establishes a sufficient condition for the existence of a saddle value.

Theorem 2.1 Let $\Lambda$ be an arbitrary non-empty set, and $\mathcal{L}: \mathbb{R}^{n} \times \Lambda \longrightarrow \mathbb{R} \cup\{+\infty\}$ be convex, proper and l.s.c. with respect to its first argument. A vector $v \in \mathbb{R}^{n}$ is a saddle ia-direction of recession of $\mathcal{L}$ relative to its first argument if and only if it is a partial ia-direction of recession of $\sup _{\lambda \in \Lambda} \mathcal{L}(\cdot, \lambda)$ and (3) holds.

Proof. "Only if".

According to Definition 2.2, for some $\bar{x} \in \mathbb{R}^{n}$ one has (5). Hence, using Lemma 1.1 we obtain

$$
\begin{aligned}
\liminf _{s \rightarrow+\infty} \sup _{\lambda \in \Lambda} \mathcal{L}(\bar{x}+s v, \lambda) & =\sup _{\lambda \in \Lambda} \inf _{x \in \mathbb{R}^{n}} \mathcal{L}(x, \lambda) \leqslant \inf _{x \in \mathbb{R}^{n}} \sup _{\lambda \in \Lambda} \mathcal{L}(x, \lambda) \\
& \leqslant \liminf _{s \rightarrow+\infty} \sup _{\lambda \in \Lambda} \mathcal{L}(\bar{x}+s v, \lambda),
\end{aligned}
$$

so $v$ is an ia-direction of recession of $\sup _{\lambda \in \Lambda} \mathcal{L}(\cdot, \lambda)$. On the other hand, in view of Lemma 1.1, for $\bar{x} \in \mathbb{R}^{n}$ satisfying (5) we obtain

$$
\begin{aligned}
\sup _{\lambda \in \Lambda} \inf _{x \in \mathbb{R}^{n}} \mathcal{L}(x, \lambda) & \leqslant \inf _{x \in \mathbb{R}^{n}} \sup _{\lambda \in \Lambda} \mathcal{L}(x, \lambda) \leqslant \liminf _{s \rightarrow+\infty} \sup _{\lambda \in \Lambda} \mathcal{L}(\bar{x}+s v, \lambda) \\
& =\sup _{\lambda \in \Lambda} \inf _{x \in \mathbb{R}^{n}} \mathcal{L}(x, \lambda),
\end{aligned}
$$

which proves (3).

"If".

Since $v$ is a partial ia-direction of recession of $\sup _{\lambda \in \Lambda} \mathcal{L}(\cdot, \lambda)$, for some $\bar{x} \in \mathbb{R}^{n}$ one has

$$
\liminf _{s \rightarrow+\infty} \sup _{\lambda \in \Lambda} \mathcal{L}(\bar{x}+s v, \lambda)=\inf _{x \in \mathbb{R}^{n}} \sup _{\lambda \in \Lambda} \mathcal{L}(x, \lambda) .
$$

Hence, using (3) we get

$$
\liminf _{s \rightarrow+\infty} \sup _{\lambda \in \Lambda} \mathcal{L}(\bar{x}+s v, \lambda)=\sup _{\lambda \in \Lambda} \inf _{x \in \mathbb{R}^{n}} \mathcal{L}(x, \lambda),
$$


which means that $v \in \mathbb{R}^{n}$ is a saddle ia-direction of recession of $\mathcal{L}$ relative to its first argument.

Our next theorem characterizes ia-directions of recession of functions in terms of the new notion of special direction of recession for bifunctions introduced in Definition 2.3.

Theorem 2.2 Let $f: \mathbb{R}^{n} \rightarrow \mathbb{R} \cup\{+\infty\}$ be an l.s.c. proper convex function. $A$ direction of recession $v$ of $f$ is an ia-direction of recession if and only if it

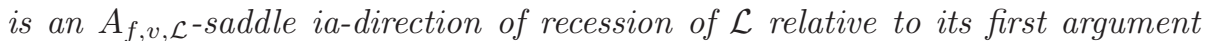
for every $\mathcal{L}: \mathbb{R}^{n} \times \Lambda \longrightarrow \mathbb{R} \cup\{+\infty\}$, with $\Lambda$ being an arbitrary non-empty set, which is convex, proper and l.s.c. with respect to its first argument and satisfies the following properties:

(a) $\sup _{\lambda \in \Lambda} \inf _{x \in \mathbb{R}^{n}} \mathcal{L}(x, \lambda) \geq \inf _{x \in \mathbb{R}^{n}} f(x)$;

(b) The set

$$
A_{f, v, \mathcal{L}}:=\left\{\bar{x} \in \mathbb{R}^{n}: \liminf _{s \rightarrow+\infty} \sup _{\lambda \in \Lambda} \mathcal{L}(\bar{x}+s v, \lambda) \leq \liminf _{s \rightarrow+\infty} f(\bar{x}+s v)\right\}
$$

is non-empty.

Proof. "Only if". Take $\bar{x} \in A_{f, v, \mathcal{L}}$. According to Definition 1.4, we have (4). Since $\bar{x} \in A_{f, v, \mathcal{L}}$, combining (a) with (4) and using Lemma 1.1, we obtain

$$
\begin{aligned}
\liminf _{s \rightarrow+\infty} \sup _{\lambda \in \Lambda} \mathcal{L}(\bar{x}+s v, \lambda) & \leq \liminf _{s \rightarrow+\infty} f(\bar{x}+s v)=\inf _{x \in \mathbb{R}^{n}} f(x) \leq \sup _{\lambda \in \Lambda} \inf _{x \in \mathbb{R}^{n}} \mathcal{L}(x, \lambda) \\
& \leq \inf _{x \in \mathbb{R}^{n}} \sup _{\lambda \in \Lambda} \mathcal{L}(x, \lambda) \leq \liminf _{s \rightarrow+\infty} \sup _{\lambda \in \Lambda} \mathcal{L}(\bar{x}+s v, \lambda),
\end{aligned}
$$

which yields (5).

"If". Let $(\mathcal{P})$ be the convex program with objective function $f$ and constraint function $g: \mathbb{R}^{n} \longrightarrow \mathbb{R}$ identically zero, and let $\mathcal{L}: \mathbb{R}^{n} \times \mathbb{R}_{+} \longrightarrow \mathbb{R} \cup\{+\infty\}$ be the Lagrangian of $(\mathcal{P})$, defined by $(2)$. Then $\mathcal{L}(\cdot, \lambda)=f$ for every $\lambda \in \mathbb{R}_{+}$; hence, $A_{f, v, \mathcal{L}}=\mathbb{R}^{n}$, and $\mathcal{L}$ satisfies the conditions in the statement. Therefore, $v$ is an $\mathbb{R}^{n}$-saddle ia-direction of recession of $\mathcal{L}$ relative to its first argument, and so for every $\bar{x} \in \mathbb{R}^{n}$ we have (5), which reduces to (4) in view of the definition of $\mathcal{L}$.

Similarly, we next characterize ia-directions of recession of sets in terms of directions of recession of bifunctions.

Theorem 2.3 Let $A$ be a non-empty closed and convex subset of $\mathbb{R}^{n}$. A direction of recession $v$ of $A$ is an ia-direction of recession if and only if for every $\mathcal{L}: \mathbb{R}^{n} \times \Lambda \longrightarrow \mathbb{R} \cup\{+\infty\}$, with $\Lambda$ being an arbitrary non-empty set, which is convex, proper and l.s.c. with respect to its first argument and such that $v$ is a direction of recession of $\mathcal{L}(\cdot, \bar{\lambda})$ for some $\bar{\lambda} \in \Lambda$ satisfying $\mathcal{L}(x, \bar{\lambda})=\sup _{\lambda \in \Lambda} \mathcal{L}(x, \lambda)$ for all $x \in A$, one has (3). 
Proof. "Only if". Let $x \in \mathbb{R}^{n}$. As $v$ is an ia-direction of recession of $A$, for $s$ large enough we have $x+s v \in A$. As $v$ is a direction of recession of $\mathcal{L}(\cdot, \bar{\lambda})$, it follows that

$$
\mathcal{L}(x+s v, \bar{\lambda}) \leqslant \mathcal{L}(x, \bar{\lambda}) \quad \forall s \geqslant 0,
$$

and so

$$
\inf _{x \in A} \mathcal{L}(x, \bar{\lambda})=\inf _{x \in \mathbb{R}^{n}} \mathcal{L}(x, \bar{\lambda}) .
$$

In view of Lemma 1.1, we obviously have

$$
\begin{aligned}
\inf _{x \in \mathbb{R}^{n}} \mathcal{L}(x, \bar{\lambda}) & \leqslant \sup _{\lambda \in \Lambda} \inf _{x \in \mathbb{R}^{n}} \mathcal{L}(x, \lambda) \leqslant \inf _{x \in \mathbb{R}^{n}} \sup _{\lambda \in \Lambda} \mathcal{L}(x, \lambda) \leqslant \inf _{x \in A} \sup _{\lambda \in \Lambda} \mathcal{L}(x, \lambda) \\
& =\inf _{x \in A} \mathcal{L}(x, \bar{\lambda}) .
\end{aligned}
$$

From relations (7) and (8) we immediately obtain (3).

"If". Let $f: \mathbb{R}^{n} \longrightarrow \mathbb{R} \cup\{+\infty\}$ be an l.s.c. proper convex function, and $g: \mathbb{R}^{n} \longrightarrow(\mathbb{R} \cup\{+\infty\})^{m}$ be a vector function with l.s.c. proper convex components. Assume that $A=\left\{x \in \mathbb{R}^{n}: g(x) \leq 0\right\}$ and that $v$ is a direction of recession of the consistent convex program $(\mathcal{P})$ defined by $(1)$. Let $\mathcal{L}: \mathbb{R}^{n} \times \mathbb{R}_{+}^{m} \longrightarrow \mathbb{R} \cup\{+\infty\}$ be the Lagrangian of $(\mathcal{P})$ defined by $(2)$. For every $x \in A$, we have

$$
\sup _{\lambda \in \mathbb{R}_{+}^{m}} \mathcal{L}(x, \lambda)=f(x)+\delta_{A}(x)=f(x)=\mathcal{L}(x, 0) .
$$

Hence, by the assumption, we obtain $(3)$, which means that $(\mathcal{P})$ has no duality gap. Therefore, by [7, Thm. 4.2], $v$ is an ia-direction of recession of $A$.

Using Theorems 2.1 and 2.2, we easily recover a zero duality gap result due to Ernst and Volle:

Corollary 2.1 [7, Thm. 5.1.(ii)] The duality gap of a consistent convex program is zero provided that at least one of its directions of recession is an ia-direction of recession of the objective function, or an ia-direction of recession of the feasible set.

Proof. Let $v$ be a direction of recession of the convex program $(\mathcal{P})$ defined by (1), which we assume to be consistent, and let $\mathcal{L}: \mathbb{R}^{n} \times \mathbb{R}_{+}^{m} \longrightarrow \mathbb{R} \cup\{+\infty\}$ and $A$ be the Lagrangian and the feasible set of $(\mathcal{P})$, respectively. Consider first the case when $v$ is an ia-direction of recession of $f$. Since $f \leq \mathcal{L}(\cdot, 0)$, condition (a) of Theorem 2.2 holds. Let $A_{f, v, \mathcal{L}}$ be the set defined by (6). From the equality $\sup _{\lambda \in \mathbb{R}_{+}^{m}} \mathcal{L}(\cdot, \lambda)=f+\delta_{A}$, it easily follows that $A \subseteq A_{f, v, \mathcal{L}}$; therefore $A_{f, v, \mathcal{L}} \neq \emptyset$ as $(\mathcal{P})$ is consistent. Thus, condition (b) of Theorem 2.2 holds, too. Therefore,

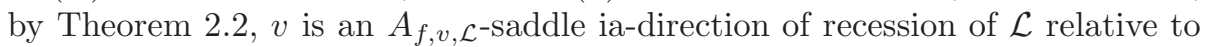
its first argument; hence, using Theorem 2.1 we obtain (3), which means that the duality gap of $(\mathcal{P})$ is zero.

Consider now the case when $v$ is an ia-direction of recession of the feasible set $A$. By Proposition 1.1, $v$ is a direction of recession of $f$ and every component 
of $g$. Hence, it is a direction of recession of $f+\delta_{\cup_{i=1}^{m} g_{i}^{-1}(\mathbb{R})}=\mathcal{L}(\cdot, 0)$; therefore, since $\mathcal{L}(x, 0)=\sup _{\lambda \in \mathbb{R}_{+}^{m}} \mathcal{L}(x, \lambda)$ for all $x \in A$, from Theorem 2.3 it follows that equality (3) holds. We thus conclude that the duality gap of $(\mathcal{P})$ is zero in this case too.

\section{Conclusions}

As proved by Ernst and Volle in [7], there exist characterizations of the objective functions and feasible sets of convex optimization problems for which the duality gap of a convex program is always zero, regardless of the constraints and, respectively, of the objective function.

In this paper, we have given a complete characterization of the existence of a saddle value for a bifunction, by considering new suitably defined notions of special directions of recession for bifunctions depending on the first argument in a convex, proper and l.s.c. way. Using Theorems 2.1 and 2.2 we obtain, as special cases, the results of Lagrangian duality theory on zero duality gap for convex programs presented in [7].

Acknowledgment. The research of the corresponding author was supported by the MICINN of Spain, Grant MTM2011-29064-C03-01, and under Australian Research Council's Discovery Projects funding scheme (project number DP140103213). He is affiliated to MOVE (Markets, Organizations and Votes in Economics).

The authors are grateful to Michel Volle for valuable remarks, which have helped us to substantially improve the manuscript.

\section{References}

1. von Neumann, J.: Über ein ökonomisches Gleichungssystem und eine Verallgemeinerung des Brouwerschen Fixpunktsatzes. Erg. eines Math. Coll., Vienna, edited by K. Menger 8, 73-83, (1937).

2. Rockafellar, R.T.: A general correspondence between dual minimax problems and convex programs. Pacific J. Math. 25(3), 597-611, (1968).

3. Simons, S.: Maximinimax, minimax, and antiminimax theorems and a result of R. C. James. Pacific J. Math. 40(3), 709-718, (1972).

4. Rockafellar, R.T.: Convex Analysis. Princeton University Press, Princeton (1970).

5. Duffin, J.R.: Clark's Theorem on linear programs holds for convex programs. Proc. Natl. Acad. Sci. USA 75(4), 1624-1626, (1978).

6. Jeyakumar, V., Wolkowicz, H.: Zero duality gaps in infinite-dimensional programming. J. Optim. Theory Appl. 67, 87107 (1990). 
7. Ernst, E., Volle, M.: Zero Duality Gap for Convex Programs: A Generalization of the Clark-Duffin Theorem. J. Optim. Theory Appl. 158(3), 668-686 (2013). 